\title{
BMJ Open Health providers' experiences with mental health specialist video consultations in primary care: a qualitative study nested within a randomised feasibility trial
}

\author{
Markus W Haun (D) , ${ }^{1}$ Mariell Hoffmann, ${ }^{1}$ Alina Wildenauer, ${ }^{1}$ Justus Tönnies (D) , ${ }^{1}$ \\ Michel Wensing (i) , ${ }^{2}$ Joachim Szecsenyi, ${ }^{2}$ Frank Peters-Klimm, ${ }^{2}$ Regina Krisam, ${ }^{3}$ \\ Dorothea Kronsteiner, ${ }^{3}$ Mechthild Hartmann, ${ }^{1}$ Hans-Christoph Friederich ${ }^{1}$
}

To cite: Haun MW, Hoffmann M, Wildenauer A, et al. Health providers' experiences with mental health specialist video consultations in primary care: a qualitative study nested within a randomised feasibility trial. BMJ Open 2021;11:e047829. doi:10.1136/ bmjopen-2020-047829

- Prepublication history and additional supplemental material for this paper are available online. To view these files, please visit the journal online (http://dx.doi.org/10.1136/ bmjopen-2020-047829).

Received 10 December 2020 Accepted 21 October 2021

\section{Check for updates}

(c) Author(s) (or their employer(s)) 2021. Re-use permitted under CC BY-NC. No commercial re-use. See rights and permissions. Published by BMJ.

For numbered affiliations see end of article.

Correspondence to Dr Markus W Haun; markus.haun@med.uniheidelberg.de

\section{ABSTRACT}

Objective Despite available effective treatments for mental health disorders, few patients in need receive even the most basic care. Integrated telepsychiatry services may be a viable option to increase access to mental healthcare. The aim of this qualitative process evaluation embedded in a randomised controlled feasibility trial was to explore health providers' experiences with a mental healthcare model integrating mental health specialist video consultations (MHSVC) and primary care.

Methods A qualitative process evaluation focusing on MHSVC in primary care was conducted. In 13 semistructured interviews, we assessed the experience of all mental health specialists, primary care physicians and medical assistants who participated in the trial. A thematic analysis, focusing on the implementation, mechanisms of impact and context, was applied to investigate the data.

Results Considering (1) the implementation, participants evaluated the consultations as feasible, easy to use and time saving. Concerning (2) the mechanisms of impact, the consultations were regarded as effective for patients. Providers attributed the patients' improvements to two key aspects: the familiarity of the primary care practice and the fast access to specialist mental healthcare. Mental health specialists observed trustful therapeutic alliances emerging and described their experience as comparable to same-room care. However, compared with same-room care, specialists perceived the video consultations as more challenging and sometimes more exhausting due to the additional effort required for establishing therapeutic alliances. Regarding (3) the intervention's context, shorter travel distances for patients positively affected the implementation, while technical failures, that is, poor Internet connectivity, emerged as the main barrier.

Conclusions MHSVCs in primary care are feasible and successful in improving access to mental healthcare for patients. To optimise engagement and comfort of both patients and health providers, future work should focus on empirical determinants for establishing robust therapeutic alliances with patients receiving MHSVC (eg, leveraging non-verbal cues for therapeutic purposes).

Trial registration number DRKS00015812; Results.
Strengths and limitations of this study

One of the first process evaluations embedded in a randomised controlled trial (RCT) evaluating mental health specialist video consultations in primary care.

- All health providers from various professions (primary care physicians, medical assistants, mental health specialists) involved in the RCT participated in the interviews.

- Data were measured prior to the onset of the COVID-19 pandemic, which may have generally increased familiarity with telemedicine of health providers.

- High participation rate in member checking (92\%) points to the credibility of the findings.

\section{INTRODUCTION}

Many patients with mental health disorders such as depression and anxiety face several challenges when seeking specialist mental healthcare. These challenges include the fear of being stigmatised and the often frustrating search for specialists caused by long waiting times for appointments ${ }^{1-3}$ and long travel distances to specialists, especially in rural and remote areas. ${ }^{4}$ Therefore, most patients turn to their primary care physician (PCP) for initial help and out of preference for the longitudinal relationship in primary care. ${ }^{5} \mathrm{It}$ is indisputable that PCPs do effectively help many patients with depression or anxiety. However, a significant number of patients in primary care, especially those with mulitmorbidity or chronic conditions, need specialist mental healthcare.

Previous work has shown that integrating mental health specialists (MHSs) in primary care increases the accessibility of specialist care and improves effectiveness outcomes. ${ }^{6}$ 
However, due to too limited resources, small and remote practices struggle to employ additional staff, for example, MHS as case managers. Since the average number of PCPs per practice in the UK, in France or in Germany (predominance of single-handed practitioners) is much lower than in the USA, for example, the barriers are even higher in those healthcare systems. ${ }^{78}$

More recent integrated care approaches feature videobased care models providing healthcare at a distance by virtually connecting patients and health providers. ${ }^{9}$ Realtime video consultations (VCs) for delivering specialist healthcare yield comparable effectiveness to same-room care. ${ }^{10-14}$ Hence, MHSVCs embedded in primary care are a promising mode to expand the reach of specialist mental healthcare. While the existing literature most commonly focuses on effectiveness outcomes, little is known on implementing MHSVCs into primary care practices from the perspective of participating health providers who serve as key players for initiating (referral of patients by primary care staff) and also delivering (MHS) successful treatments. ${ }^{15}$

The PROVIDE (ImPROving cross-sectoral collaboration between primary and psychosocial care: An implementation study on VIDEo consultations, https://www. provide-project.de/ziel-konzept/?lang=en) project aims to improve cross-sectoral collaboration between primary and psychosocial care by managing depression and anxiety disorders in primary care through MHSVCs. Following an implementation science paradigm, PROVIDE promotes the uptake of telepsychiatry into routine care to improve the quality and effectiveness of primary care mental health. ${ }^{16}$ Prior to embarking on a sufficiently powered confirmatory trial, the intervention was evaluated in a feasibility trial (PROVIDE-B). ${ }^{17} 18$ The purpose of this qualitative process evaluation embedded in the feasibility trial was to understand the functioning of the intervention considering the three main aspects of the Medical Research Council (MRC) guidance for process evaluation of complex interventions, that is, (1) how MHSVCs were implemented into daily practice and, if applicable, which adaptions were necessary (implementation), (2) the impact of MHSVCs on patient outcomes from the perspective of all health providers involved in the delivery of the intervention (mechanisms of impact) and (3) the contextual barriers and facilitators shaping the implementation of MHSVCs (context). ${ }^{19} 20$

\section{METHODS}

\section{Study design}

We conducted a qualitative process evaluation of the PROVIDE-B randomised controlled feasibility trial with all health providers involved and with patients who received the MHSVCs. Findings for patients will be reported in a separate paper. In the work presented here, we intended to explore health providers' experiences with the delivery of the intervention. We took a critical realist position when designing the study, analysing the data and interpreting the findings. We followed the MRC guidance for process evaluation of complex interventions and the Consolidated criteria for Reporting Qualitative research guidelines for reporting qualitative research (online supplemental file 1). ${ }^{19} 21$ All procedures performed in the study involving human participants were in accordance with the ethical standards of the institutional research committee and with the 1964 Declaration of Helsinki and its later amendments and it pre-registered with the German Clinical Trials Register.

\section{Setting}

The trial was run in five primary care practices in the South Germany. The intervention comprised real-time VCs conducted on a secure, web-based videoconferencing platform on a subscription basis (arztkonsultation ak GmbH, https://arztkonsultation.de) between patients with depression and/or anxiety in a designated room in their primary care practices and MHSs located off-site. The intervention included establishing a trustful therapeutic alliance between patients and MHSs, systematic diagnostics and, if indicated, brief solution-focused therapy. Patients in the intervention group received up to five 50 min MHSVCs in biweekly intervals. After the last MHSVC, the MHSs sent a written case summary to the PCPs on which follow-up care pursued by PCPs was based. Trial outcomes focused on feasibility, patient-reported outcomes and cost-effectiveness. The trial design and the intervention are described in the study protocol for the feasibility ${ }^{17}$ and the main trial, respectively ${ }^{22}$ as well as in the intervention manual (https://bit.ly/32VAvI9).

\section{Participants and recruitment}

We contacted all MHSs, PCPs and medical assistants participating in the PROVIDE-B trial by telephone and invited them for a one-off interview. Participating in the interviews was not a prerequisite for participating in the randomised controlled feasibility trial as such. We did not offer any individual monetary compensation for interview participation. Eventually, we conducted semistructured interviews with all 13 health providers involved in the trial, namely, with 3 MHSs, 6 PCPs and 4 medical assistants on their experiences with MHSVCs.

\section{Data collection}

We developed semistructured interview guides adapted to the professional background of the interviewees (online supplemental file 2A-C). We reviewed interview guides for all professions after the first interview but did not make any substantial changes as the guides proved coherent and comprehensive. Study objectives and data protection were made transparent to all participants who all provided written consent prior to data collection. From July to September 2019, MaH conducted individual face-to-face interviews with the PCPs (median: $42 \mathrm{~min}$, IQR: $24 \mathrm{~min}$ ) and the medical assistants (median: $17 \mathrm{~min}$, IQR: $4 \mathrm{~min}$ ) at the PCPs' practices and telephone interviews with the MHSs (median: $44 \mathrm{~min}$, IQR: $7 \mathrm{~min}$ ). $\mathrm{MaH}$ 
was not involved in the design and conduct of the feasibility trial. We audio recorded all interviews and uploaded them to a secure server of Heidelberg University Hospital, which was only accessible to the research team. In total, we conducted 12 interviews as two PCPs worked in the same practice and requested to be interviewed together. Additionally, a study nurse- the only person present besides the participant(s) and the researcher-made field notes during the interviews. We collected sociodemographic characteristics at the end of each interview. We did not repeat any interviews.

\section{Data analysis}

We transcribed interviews verbatim, but refrained from returning them to the participants for comment to minimise participant burden. Two members of the research team (MaH and $\mathrm{AW}$, female, master's degree in sociology, research assistant) independently performed an inductive/data-driven thematic analysis prior to knowing the trial outcomes. ${ }^{202324}$ Both coders were neither involved in the delivery of the intervention nor in the quantitative analysis of the trial. First, both coders independently analysed one transcript of each provider group inductively in MAXQDA Analytics Pro 2020 by developing initial codes, including themes that capture important feasibility components. Second, they compared their themes and developed a joint coding system. Third, both coders discussed and agreed on a code system which they together with MWH reviewed for consistency of the developed themes. Fourth, MaH applied the final coding system (online supplemental file 3) to the remaining transcripts and added newly emerging subcodes in consultation with AW and MWH. Theme saturation was reached when the data did not provide any new themes or meaning of themes, that is, when the themes covered all the data. ${ }^{25}$ Finally, we assigned the inductively developed themes to the key aspects of process evaluations. Process evaluations attempt to document how an intervention is implemented and what was actually delivered, compared with that intended to be delivered.$^{26}$ According to the MRC guidance, key aspects of process evaluations for complex interventions are (a) implementation (ie, application of interventions), (b) mechanisms of impact (of the interventions and strategies to implement these) and (c) context (ie, factors in the setting and target group associated with implementation and impact). ${ }^{19}$

\section{Member checking}

To ensure the accuracy and credibility of our findings, we asked the interviewees for communicative validation of the findings. ${ }^{27}$ We sent an anonymised written summary of all findings to all participants and asked them to review as to whether these findings reflect their experiences. Subsequently, we called all participants to gather their feedback and refine our results.

\section{Patient and public involvement}

To inform the development of the research question by patients' priorities and preferences, we conducted a preimplementation qualitative study drawing on interviews with patients presenting to the outpatient clinic of the Department of General Internal Medicine and Psychosomatics at Heidelberg University Hospital, Heidelberg, Germany. ${ }^{28}$ Specifically, we tailored the intervention accordings to patients' needs based on the preimplementation findings. In the study presented here, we did not involve patients in the recruitment, as the sampling frame for the health providers resulted from the trial. However, we dissmenitated the results to the participating health providers as part of member checking. Burden of the intervention for patients will be reported in the separate paper on the findings from interviews with participating patients.

\section{RESULTS}

We present our findings for the three key aspects of a process evaluation (implementation, mechanisms of impact and context), followed by the results from member checking.

\section{Sample}

Table 1 presents the sociodemographic characteristics of the sample.

\section{Implementation: application of MHSVCs}

Considering the implementation, we investigated how MHSVCs were conducted and implemented into daily practice and if adaptions were necessary.

All providers discussed their practical experiences with MHSVCs, especially its technical fesasibility. Most providers noticed interruptions of the audio and/or visual transmission, while the handling of the device, such as a tablet, and the videoconferencing platform was perceived as easy by both patients and providers:

[The handling worked out] Very well. We had younger and also older patients, but both groups handled it well [the MHSVC] (medical assistant number 3).

When difficulties occurred, they were quickly overcome. For example, one medical assistant stated that some patients felt uncomfortable holding the tablet in their hands, which was solved by providing a tablet stand. Moreover, some health providers mentioned additional tangible implementation barriers such as poor acoustics in the designated room in the primary care practice or tablet screens deemed too small.

MHSs were divided on the appropriate total number of MHSVC sessions per patient. Two MHSs were convinced that a fixed number of five sessions was sufficient to provide basic support and triage in addition to diagnostics, but still made it possible to see a large number of patients introducing themselves with depression and/or anxiety to the PCP. This notion fitted well with a population health perspective accounting for both reach and 
Table 1 Characteristics for health provider interviews

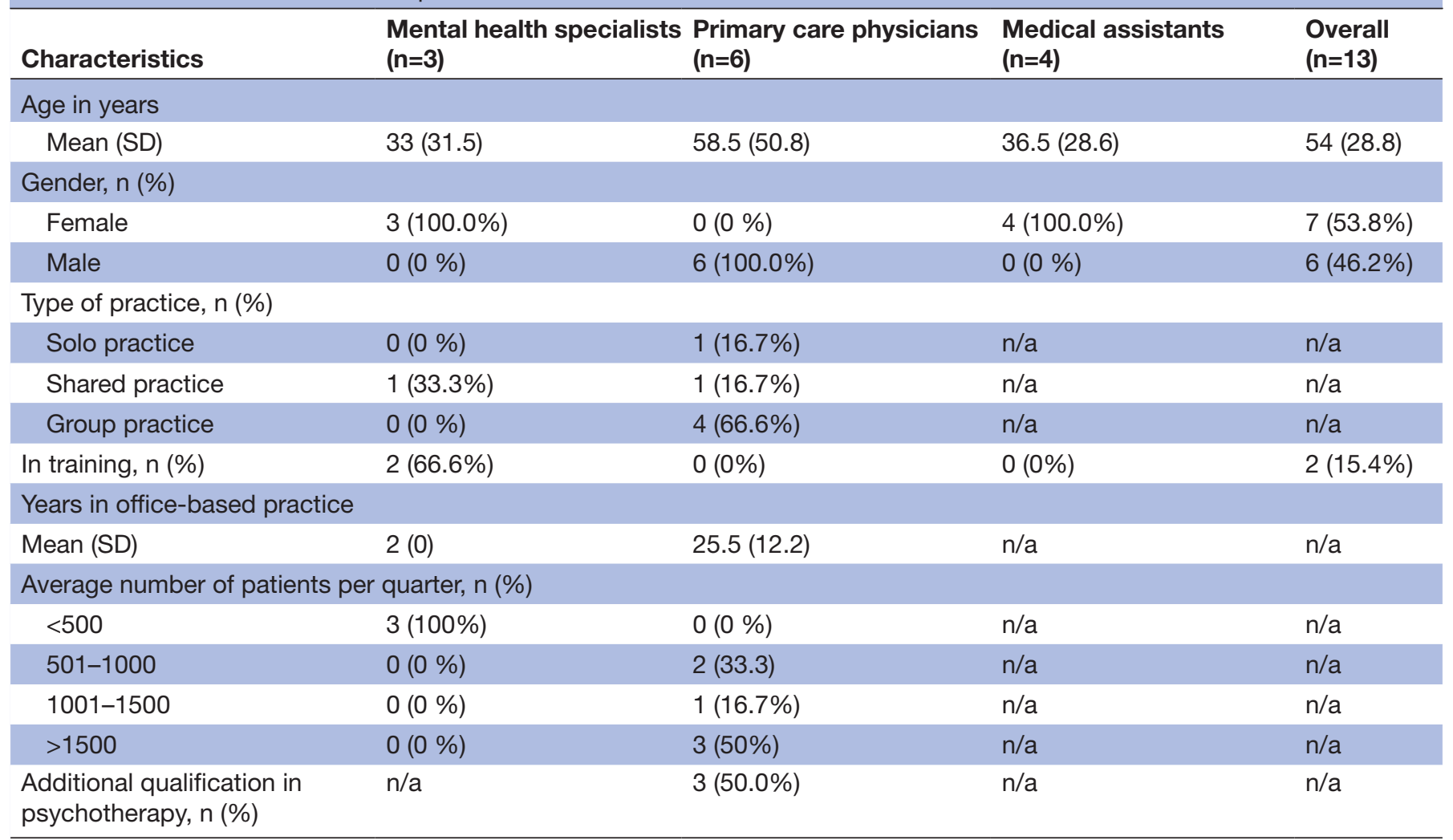

n/a, not applicable.

effectiveness. In contrast, one MHS advocated for a flexible number of sessions tailored to the individual patient arguing that patients with severe symptoms might need more than five sessions.

All participants from the primary care practices evaluated the integration into daily practice as easy and feasible. Taking into account the tightly organised dayto-day routine in the practice, some even expected that being able to rapidly offer MHSVCs to burdened patients would save the primary care team frustrating attempts to refer patients and ultimately a notable amount of time:

Just knowing that the therapy would start soon was a relief. It is no use if I give someone a list [of officebased mental health specialists], [...] and then he won't get an appointment and faces another obstacle in his life. And [...] needs even more help (PCP number 4).

In particular, the appointment management was perceived to be very easy and well organised. MHS and patients agreed on an appointment for the next MHSVC and the MHS sent the appointment to the general practice and the study team. Communication about appointments was mainly between the MHS and the medical assistants. The PCPs were rarely involved.

She [the MHS] always sent me e-mails about when the next ones [MHSVCs] would take place [...]. The time slots were agreed upon at the beginning.
The patients then came in and at the first session I explained everything to them and then they did it themselves. If there were small problems, I went back in and helped. That all worked out very well (medical assistant number 4 ).

To save even more time and spatial resources, one PCP preferred that the MHSVCs be conducted from the patients' home after the first session in the primary care practice. Focussing on patients, most PCPs perceived their acceptance of MHSVCs generally as high. Of the patients struggling with MHSVCs, according to the primary care staff, a minority was concerned about the lack of sameroom contact, while the majority entertained reservations about psychotherapy as such.

Regarding the collaboration between the involved health providers, the final case summary written by the MHS for the PCP emerged as the main facilitator. One MHS regularly called the PCP to decide on the patients' follow-up treatment. However, most participants did not directly collaborate with their professional counterparts referring to the tightly organised day-to-day routine of the primary care practice as a systematic barrier. Suggestions for adaptions, put forward by the PCPs, included a written diagnostic feedback from the MHSs early in the course of the consultations:

[If I got a] written, one-sided feedback how she [the MHS] sees it, I would find it as least worthwhile, [... 
[for example] she also sees it as I do or maybe has another idea that I didn't think of (PCP number 6).

Somewhat in contrast, MHSs clearly adovacted for personal contact, for example, to hand the patient over to the PCP after the last VC:

I would actually wish for a phone call with the PCP at the end of the MHSVC where he asks something like "I referred her to you because of this and that condition, what happened to her?" and one would answer" We worked on this and that and I would advise you, for the patient's sake, to pay attention to this and that" (MHS number 3).

Clearly, from their perspective, the written case summary prescribed in the intervention was not sufficient, but informal contact was required to foster collaboration between health providers more strongly.

\section{Mechanisms of impact of mental health VCs}

To examine the impact of MHSVCs from the perspective of all providers involved in their delivery, we aimed to identify how the intervention worked and which aspects of the MHSVCs contributed to their effectiveness.

All participants stated that the vast majority of patients benefited from the model, highlighting its provision of fast access to specialist mental healthcare as a key factor:

I have the feeling that they [the patients] benefit from the fact that someone will soon listen to them [...] So I noticed small changes in all patients, especially a form of relief (MHS number 3 ).

Health providers observed that patients were relieved from the frustrating seeking for available specialists and long waiting times and instead provided with care that was 'easy and comfortable' (PCP number 5). Some PCPs mentioned a second key factor contributing to patients' benefit, namely, the familiar environment of the primary care practice:

Patients certainly benefitted from the MHSVC, because it [the primary care practice] was a familiar environment. [...] This is certainly an advantage (medical assistant number 1).

The primary care practice also provided low-threshold access for people concerned with being stigmatised by seeking support from specialist mental healthcare. In fact, patients often had long-standing, grown, and trusting relationships with the practice team and, hence, could give the MHS a leap of faith when starting their VCs:

For some [patients], I think it was helpful that that it took place in the primary care practice, since it provided a certain familiarity [...] I would go so far as to say that most people would not have logged in from home. [...] and because they trust him [the PCP], the specialist was also trusted and therefore the consultations were easy to implement (MHS number 1).
For the MHSs, it was essential that a sustainable therapeutic relationship could also be established in VCs. Indeed, specialists perceived the emerging therapeutic alliances as comparable to conventional same-room care:

I am surprised, but the computer is obviously no obstacle. Sometimes I feel a sense of distance, but I don't think that applies to patients, at least not to some (MHS number 3).

I think it is not necessary to be physically in the same room to create a certain emotional closeness with the patient (MHS number 2).

Nevertheless, both PCPs and MHSs also mentioned barriers that affected the impact of the VCs. In their view, the missing same-room contact made it rather difficult for them to pick up and adress body language expressions, for example, facial expressions or gestures:

I doubt that feelings can be fully communicated or that real instructions can be achieved compared to face-to-face conversations. It may not always be decisive, but it is a real disadvantage (PCP number 1 ).

Some MHSs suspected that this limitation could have reduced the effectiveness of the consultations in patients with severe symptoms. The MHSs also indicated that they had made additional efforts to be fully attentive and empathetic to the patient to compensate for the imminent loss of non-verbal cues relevant for the therapy. Few MHSs experienced the consultations as more strenuous and as an overall more fragile setting compared with same-room care. They related fatigue to the connectivity failures and to the fact that patients seemed to be less committed to this type of therapy and, in turn, the MHSs were more concerned with the possibiltiy of patients discontinuing the therapy. In this regard, some MHSs felt more confident und comfortable with same-room interaction.

Context: factors in the setting associated with implementation and impact

To understand how the context affected the delivery of MHSVC, interviewees were asked about the relevance of external factors affecting the implementation.

Overall, context was less frequently mentioned compared with the other two key aspects addressed in the process evaluation. Regarding tangible requirements for the primary care practices, some health providers saw the expansion of the bandwith for a better connectivity as indispensable for enhancing video, audio and, ultimately, the quality of the intervention. Few health providers suggested that the room designated for the consultations should be subjected to a thorough standardised check for sound insulation. Beneficial facilitators outside the intervention as such included, according to some health providers, shorter travel distances for the patients and, to some extent, workload relief for the PCPs who were enabled to refer patients with complex mental health issues or patients with physical and mental health comorbidity successfully. 


\section{Member checking}

Twelve of the 13 participants (92\%) took part in member checking and confirmed that the final consolidation of the results adequately reflected their personal experiences with MHSVCs. Some participants added suggestions for improving the model, that is, by providing a larger sized tablet screen or increasing the sound quality. One MHS firmly disagreed with the statement that MHSVCs come with a lower threshold for patients to abruptly discontinue therapy, underscoring that she perceived patients to be highly committed to these consultations.

\section{DISCUSSION}

This study aimed to explore health providers' initial experiences with implementing MHSVCs in primary care for managing patients with depression and anxiety. We found that, considering (1) the implementation, most participants stated that the integration of the VCs into the daily routine of the primary care practices was not only feasible but potentially time saving for the practice staff, particularly when attending to patients with complex mental health issues or patients with physical and mental health comorbidity. Concerning (2) the mechanisms of impact, two key factors contributing to patients' benefit emerged, namely, faster access to specialist mental healthcare and the familiar environment of the primary care practice with the latter functioning as a destigmatising facilitator. The MHSs observed trustful therapeutic alliances developing and described their experience as comparable to same-room care. However, compared with same-room care, specialists perceived the $\mathrm{VC}$ as more challenging and sometimes more exhausting due to the additional effort required for establishing therapeutic alliances. Regarding (3) the intervention's context, we identified connectivity and privacy of the room designated for the consultations as main tangible requirements. Beneficial facilitators outside the intervention as such comprised less travel efforts for the patients and workload relief for the primary care practice staff.

Prior investigations on MHSVCs mostly focus on efficiency, satisfaction and attitudes towards VCs before and after its use ${ }^{29} 30$ and have demonstrated that VCs in primary care can be time saving for patients and that clinicians usually underscore the importance of an existing doctor-patient relationship prior to VCs. Our study adds that, given the rapid onset of the MHSVCs and easy implementability, they can also save time for providers themselves in the tightly organised day-to-day routine of PCPs' practices. Moreover, the grown relationships that patients entertained with the practice team enabled the patients to give the MHSs a leap of faith when starting their VCs. Since building up to a solid therapeutic alliance is the main tenet particularly for online interventions, this leap of faith may constitute a key facilitator for the MHSVCs and, ultimately, beneficial patient outcomes. ${ }^{28}$ 31-36

For the MHSs, the VCs required more concentration and finally demanded a greater effort from them compared with same-room care. While this has been previously attributed to the handling of the hardware and software as well as connectivity failures, ${ }^{31} 33$ 37-39 our study adds that compensating for the limited access to non-verbal cues during VCs constitutes another key factor for health providers' fatigue. At any rate, there is some evidence for a mounting gap between the empirical support for conventional integrated care approaches and the implementation of these models. ${ }^{36}$ Thus, faster access to specialist mental healthcare has been promoted as a central asset of VCs for a long time. ${ }^{3740-43}$

This study has some limitations. First, data were collected before the COVID-19 pandemic began-at a time when VCs were the exception rather than the rule in healthcare, at least in Germany. In the wake of the COVID-19 pandemic, VCs are increasingly widely used in routine care. ${ }^{44-46}$ However, while this might have changed clinicians familiarity with VCs, integrated care models for primary care mental health as such are still rarely implemented. Second, the MHSs had no prior clinical experience with VCs, which might have contributed to a more cautious attitude and concerns with the lack of same-room contact. ${ }^{47}$ Hence, the next step to further elucidate the intertwining of therapeutic alliance and virtual contact would be to compare data from experienced and nonexperienced MHSs. Third, we did not engage any payors, who play a key role when it comes to the incorporation of healthcare innovations into routine care but will do so when effectiveness outcomes from the fully powered, confirmatory RCT will be available. The present findings do provide a detailed insight into the implementation of video-based integrated mental healthcare from the perspective of the stakeholders most directly involved in the intervention delivery. The credibility of the findings is clearly supported by the high level of participation in member checking, which indicated a broad match of the findings with the individual experiences.

\section{CONCLUSIONS}

Health providers report that MHSVCs in primary care are feasible and successful in improving access to mental healthcare for patients. To optimise telepsychiatry services and facilitate the engagement and comfort of both patients and health providers with MHSVC, future work should focus on empirical determinants for establishing robust therapeutic alliances with patients receiving VCs. Based on our findings, it seems specifically promising to better understand how MHSs can best pick up non-verbal cues and use them for therapeutic purposes. ${ }^{48}$ With respect to more tangible aspects, poor connectivity as a main barrier has to be targeted systematically to enable the broad and sustainable use of telepsychiatry services for primary care services, particularly in rural and remote areas. Given that our qualitative results from the feasibility trial point to clinical benefits for patients, we have embarked on a full-scale pragmatic trial assessing the clinical and cost-effectiveness of MHSVCs. 
Author affiliations

${ }^{1}$ Department of General Internal Medicine and Psychosomatics, Heidelberg University, Heidelberg, Baden-Württemberg, Germany

${ }^{2}$ Department of General Practice and Health Services Research, Heidelberg University, Heidelberg, Baden-Württemberg, Germany

${ }^{3}$ Institute of Medical Biometry and Informatics, Heidelberg University, Heidelberg, Baden-Württemberg, Germany

Acknowledgements The authors declare that there is no conflict of interest. Financial support for this study was provided entirely by a grant from the German Federal Ministry of Education and Research (BMBF) (grant number 01GY1612). The funding agreement ensured the authors' independence in designing the study, interpreting the data, writing, and publishing the report. The funder was not actively involved in conduct of the study.

Contributors All authors participated in one or more aspects of the study design and execution. MWH (guarantor), JT, MW, JS, FP-K, MeH and H-CF designed the study. MaH conducted the interviews. MaH, AW and MWH performed the initial data analysis. MWH, MaH and AW reached consensus on the initial findings. Emergent codes and supporting quotes, identified by MaH and AW, were checked, adapted, and themes developed iteratively through feedback provided by MWH, JT, MeH. All authors (MWH, MaH, AW, JT, MW, JS, FP-K, RK, DK, MeH and H-CF) were involved in writing and reviewing the manuscript for publication. All authors approved the version of the manuscript to be published and have agreed to be accountable for all aspects of the work

Funding This work was supported entirely by the German Federal Ministry of Education and Research (BMBF) (grant number 01GY16129). The funding agreement ensured the authors' independence in designing the study, interpreting the data, writing and publishing the report.

\section{Competing interests None declared.}

Patient consent for publication Not applicable.

Ethics approval This study was approved by the Ethics Committee of the Medical Faculty Heidelberg, Heidelberg University (number S-634/2018).

Provenance and peer review Not commissioned; externally peer reviewed.

Data availability statement Data are available upon reasonable request.

Supplemental material This content has been supplied by the author(s). It has not been vetted by BMJ Publishing Group Limited (BMJ) and may not have been peer-reviewed. Any opinions or recommendations discussed are solely those of the author(s) and are not endorsed by BMJ. BMJ disclaims all liability and responsibility arising from any reliance placed on the content. Where the content includes any translated material, BMJ does not warrant the accuracy and reliability of the translations (including but not limited to local regulations, clinical guidelines, terminology, drug names and drug dosages), and is not responsible for any error and/or omissions arising from translation and adaptation or otherwise.

Open access This is an open access article distributed in accordance with the Creative Commons Attribution Non Commercial (CC BY-NC 4.0) license, which permits others to distribute, remix, adapt, build upon this work non-commercially, and license their derivative works on different terms, provided the original work is properly cited, appropriate credit is given, any changes made indicated, and the use is non-commercial. See: http://creativecommons.org/licenses/by-nc/4.0/.

\section{ORCID iDs}

Markus W Haun http://orcid.org/0000-0003-1851-3747

Justus Tönnies http://orcid.org/0000-0002-0308-8226

Michel Wensing http://orcid.org/0000-0001-6569-8137

\section{REFERENCES}

1 Cunningham PJ. Beyond parity: primary care physicians' perspectives on access to mental health care. Health Aff 2009;28:w490-501.

2 Wang PS, Lane M, Olfson M, et al. Twelve-month use of mental health services in the United States: results from the National comorbidity survey replication. Arch Gen Psychiatry 2005;62:629-40.

3 Thornicroft G, Chatterji S, Evans-Lacko S, et al. Undertreatment of people with major depressive disorder in 21 countries. $\mathrm{Br}$ Psychiatry 2017;210:119-24.

4 Greenhalgh T, Shaw S, Wherton J, et al. Real-world implementation of video outpatient consultations at macro, meso, and micro levels: mixed-method study. J Med Internet Res 2018;20:e150.
5 Gaebel W, Kowitz S, Fritze J, et al. Use of health care services by people with mental illness: secondary data from three statutory health insurers and the German statutory pension insurance scheme. Dtsch Arztebl Int 2013;110:799-808.

6 Fortney JC, Pyne JM, Turner EE, et al. Telepsychiatry integration of mental health services into rural primary care settings. Int Rev Psychiatry 2015;27:525-39.

7 Schoen C, Osborn R, Doty MM, et al. A survey of primary care physicians in eleven countries, 2009: perspectives on care, costs, and experiences. Health Aff 2009;28:w1171-83.

8 Kringos DS, Boerma WGW, Hutchinson A. Building primary care in a changing Europe case studies, 2015. Available: http://www.euro. who.int/en/about-us/partners/observatory/publications/studies/ building-primary-care-in-a-changing-europe-case-studies [Accessed 31 May 2020].

9 Salisbury C, O'Cathain A, Edwards L, et al. Effectiveness of an integrated telehealth service for patients with depression: a pragmatic randomised controlled trial of a complex intervention. Lancet Psychiatry 2016;3:515-25.

10 Backhaus A, Agha Z, Maglione ML, et al. Videoconferencing psychotherapy: a systematic review. Psychol Serv 2012;9:111-31.

11 Gonzalez GE, Brossart DF. Telehealth videoconferencing psychotherapy in rural primary care. Journal of Rural Mental Health 2015;39:137-52.

12 Hilty DM, Ferrer DC, Parish MB, et al. The effectiveness of telemental health: a 2013 review. Telemed J E Health 2013;19:444-54.

13 Berryhill MB, Culmer N, Williams N, et al. Videoconferencing psychotherapy and depression: a systematic review. Telemed J E Health 2019;25:435-46.

14 Berryhill MB, Halli-Tierney A, Culmer N, et al. Videoconferencing psychological therapy and anxiety: a systematic review. Fam Pract 2019;36:53-63

15 Fletcher TL, Hogan JB, Keegan F, et al. Recent advances in delivering mental health treatment via video to home. Curr Psychiatry Rep 2018;20:56

16 Bauer MS, Damschroder L, Hagedorn H, et al. An introduction to implementation science for the non-specialist. BMC Psychol 2015;3:32

17 Tönnies J, Hartmann M, Wensing M, et al. Mental health specialist video consultations for patients with depression or anxiety disorders in primary care: protocol for a randomised controlled feasibility trial. BMJ Open 2019;9:e030003.

18 Tönnies J, Hartmann M, Wensing M, et al. Mental health specialist video consultations versus treatment-as-usual for patients with depression or anxiety disorders in primary care: randomized controlled feasibility trial. JMIR Ment Health 2021;8:e22569.

19 Moore GF, Audrey S, Barker M, et al. Process evaluation of complex interventions: medical research council guidance. BMJ 2015;350:h1258.

20 O'Cathain A. A practical guide to using qualitative research with randomized controlled trials. Oxford University Press, 2018.

21 Tong A, Sainsbury P, Craig J. Consolidated criteria for reporting qualitative research (COREQ): a 32-item checklist for interviews and focus groups. Int J Qual Health Care 2007;19:349-57.

22 Haun MW, Tönnies J, Brinster R. Mental health specialist video consultations versus treatment as usual in patients with depression or anxiety disorders in primary care: study protocol for an individually randomised superiority trial (the provide-C trial). Trail 2021;22.

23 Braun V, Clarke V. Using thematic analysis in psychology. Qual Res Psychol 2006;3:77-101.

24 Oakley A, Strange V, Bonell C, et al. Process evaluation in randomised controlled trials of complex interventions. $B M$ 2006;332:413-6.

25 Hennink MM, Kaiser BN, Marconi VC. Code saturation versus meaning saturation: how many interviews are enough? Qual Health Res 2017;27:591-608

26 London School of Hygiene \& Tropical Medicine. Process evaluation. Available: https://www.Ishtm.ac.uk/research/centres/centreevaluation/process-evaluation [Accessed 1 Sep 2021]

27 Birt L, Scott S, Cavers D, et al. Member checking: a tool to enhance trustworthiness or merely a NOD to validation? Qual Health Res 2016;26:1802-11.

28 Bleyel C, Hoffmann M, Wensing M, et al. Patients' perspective on mental health specialist video consultations in primary care: qualitative preimplementation study of anticipated benefits and barriers. J Med Internet Res 2020;22:e17330.

29 Hammersley V, Donaghy E, Parker R, et al. Comparing the content and quality of video, telephone, and face-to-face consultations: a non-randomised, quasi-experimental, exploratory study in UK primary care. Br J Gen Pract 2019;69:e595-604. 
30 Donaghy E, Atherton H, Hammersley V, et al. Acceptability, benefits, and challenges of video consulting: a qualitative study in primary care. Br J Gen Pract 2019;69:e586-94.

31 Cowan KE, McKean AJ, Gentry MT, et al. Barriers to use of telepsychiatry: clinicians as gatekeepers. Mayo Clin Proc 2019;94:2510-23.

32 Hoffmann M, Hartmann M, Wensing M, et al. Potential for integrating mental health specialist video consultations in office-based routine primary care: cross-sectional qualitative study among family physicians. J Med Internet Res 2019;21:e13382.

33 Hoffmann M, Wensing M, Peters-Klimm F, et al. Perspectives of psychotherapists and psychiatrists on mental health care integration within primary care via video consultations: qualitative Preimplementation study. J Med Internet Res 2020;22:e17569.

34 Topooco N, Riper H, Araya R, et al. Attitudes towards digital treatment for depression: a European stakeholder survey. Internet Interv 2017;8:1-9.

35 Sucala M, Schnur JB, Constantino MJ, et al. The therapeutic relationship in e-therapy for mental health: a systematic review. $J$ Med Internet Res 2012;14:e110.

36 Ion A, Sunderji N, Jansz G, et al. Understanding integrated mental health care in "real-world" primary care settings: What matters to health care providers and clients for evaluation and improvement? Families, Systems, \& Health 2017;35:271-82.

37 Christensen LF, Moller AM, Hansen JP, et al. Patients' and providers' experiences with video consultations used in the treatment of older patients with unipolar depression: a systematic review. J Psychiatr Ment Health Nurs 2020;27:258-71.

38 Randhawa RS, Chandan JS, Thomas T, et al. An exploration of the attitudes and views of general practitioners on the use of video consultations in a primary healthcare setting: a qualitative pilot study. Prim Health Care Res Dev 2019;20:e5.
39 Harst L, Timpel P, Otto L, et al. Identifying barriers in telemedicinesupported integrated care research: scoping reviews and qualitative content analysis. J Public Health 2020;28:583-94.

40 Connolly SL, Miller CJ, Lindsay JA, et al. A systematic review of providers' attitudes toward telemental health via videoconferencing. Clinical Psychology: Science and Practice 2020;27:e12311.

41 Deen TL, Fortney JC, Schroeder G. Patient acceptance of and initiation and engagement in telepsychotherapy in primary care. Psychiatr Serv 2013;64:380-4.

42 Johansson AM, Lindberg I, Söderberg S. Healthcare personnel's experiences using video consultation in primary healthcare in rural areas. Prim Health Care Res Dev 2017;18:73-83.

43 Interian A, King AR, St Hill LM, et al. Evaluating the implementation of home-based videoconferencing for providing mental health services. Psychiatr Serv 2018;69:69-75.

44 Kannarkat JT, Smith NN, McLeod-Bryant SA. Mobilization of telepsychiatry in response to COVID-19-moving toward 21st century access to care. Adm Policy Ment Health 2020:1-3.

45 Mann DM, Chen J, Chunara R, et al. COVID-19 transforms health care through telemedicine: evidence from the field. J Am Med Inform Assoc 2020;27:1132-5.

46 Wind TR, Rijkeboer M, Andersson G, et al. The COVID-19 pandemic: The 'black swan' for mental health care and a turning point for ehealth. Internet Interv 2020;20:100317.

47 Barton PL, Brega AG, Devore PA, et al. Specialist physicians knowledge and beliefs about telemedicine: a comparison of users and nonusers of the technology. Telemed J E Health 2007;13:487-500.

48 Fisher S, Guralnik T, Fonagy P, et al. Let's face it: video conferencing psychotherapy requires the extensive use of ostensive cues. Couns Psychol Q 2020;0:1-17. 